\title{
The Phase Theory towards the Unification of the Forces of Nature the Heart Beat of the Universe
}

\author{
${ }^{1}$ M. E. Isma'eel, ${ }^{2}$ Sherif M. E. Ismaeel \\ ${ }^{I}$ Formerly; Deputy Manager of the Armed Forces Technical Research Center of Egypt \\ ${ }^{2}$ Dr. Sherif is a lecturer with Ain Shams University Faculty of Science, Department of Physics, Cairo - Egypt
}

\begin{abstract}
A new theory has been presented, for the first time, called the "Phase Theory", which is the natural evolution of the physical thought and is considered the one beyond the super string theory. This theory solves the unsolved problems of the mysterious of matter, antimatter and interactions and makes a wide step towards the unification of the forces of nature. In this theory, the vibrating string of different frequency modes which determines the different types of elementary particles is replaced by a three dimensional infinitesimal pulsating (black)holes with the same frequency. Different types of elementary particles are determined by different phase angles associated with the same frequency. This allows the force of interactions to take place among elementary particles, without the need to invoke the notion of the force carrier particles, as the (stable) force of interactions can never take place between elementary particles at different frequencies. Besides the strong mathematical proofs given in this paper to prove its truthfulness, an experimental prediction has been given to confirm the theory presented in the form of the relation between the electron radius and quarks radii. The paper shows that quarks are direct consequence of this theory, and solves "the flavor problem" in $Q C D$, and gives the clue to answer the questions of "Why are there so many flavors? The paper also derives the equation of the big bang theory which describes the singularity of the moment of creation of the universe.
\end{abstract}

\section{Introduction}

References [1-2] give three different and distinct experimental proofs to confirm the existence of the new phenomenon of the vertical aether flow into any mass including any fundamental building block (i.e. including any elementary particle).So far, Physicists believe that, it is a common mistake to try to explain both gravity and electromagnetism using a single medium. Any attempts to do so have foundered on the grounds of inability to account for why gravity is mutually attractive and why electromagnetism is mutually repulsive. However, this problem, as will be seen later, will easily be solved by introducing, for the first time, the "the Phase Theory" which based on unidirectional vertical aether flow, as it has been formerly proved [1-2], if we take the phase angle of the flow into consideration. The concept of aether existence gives us the privilege to explain all particles in nature as well as their interactions, in terms of one simple phenomenon, i.e. the vertical Aether motion, without the necessity to invoke the notion of the force carrier particles, named, Bosons. The phase theory treats all the particles of nature at the same level. All particles have the same pulsating frequency, as the (stable) force of interactions can never take place at different frequencies. Different particles are characterized by different phase angles associated with the same frequency. The phase angle defines the type of particle as well as the type of interactions between particles, attraction, repulsion or none. The force will be repulsive, attractive or none depending on cosine the angle of the phase difference between the pulsating elementary particles, the phase difference usually called the power angle. Also, the phase theory simply interprets the force exchange by merely a "to and fro" motion of the vertical aether flow between elementary particles. And, in turn, the mass is merely a direct consequence of the aether drag force. Thus the "Phase Theory" is considered the shortest way to unify the forces of nature. Or simply, it is the theory that unifies all the forces of nature at room temperature. The paper also shows that quarks are a direct consequence of the phase theory. A sort of prediction, the paper gives the relation between the electron radius and the radii of the different types of quarks (including the top quark). It also derives an equation for the big bang theory which describes the singularity of the moment of creation of the universe. Finally, the paper contributes toward solving "the flavor problem" in QCD, and gives the clue to answer the questions of "Why are there so many flavors?

One final note, all the trials to animate the force of interactions between particles, in terms of the force carrier particles, fails to interpret the two types of forces in the same time. For example, the basketball animation between two players is a very crude analogy since it can only explain repulsive forces and gives no hint of how exchanging particles can result in attractive forces. 


\section{Basic postulates}

In the following three postulates, subjected to the verifiability principle, have been introduced to facilitate the explanation of the theories presented in this paper. Noting that "Paradigm shift in the human thought" usually takes decades to become settled and accepted.

Postulate No. 1: Our universe has no boundaries or edges either at the micro or the macro scales.

Postulate No. 2: Elementary particles are pulsating 3-dimensional sinks with definite frequency and phase where aether flows vertically into them, and they have no lower bound as it follows from Postulate I.

Postulate No. 3: (Stable) Particle interactions can never take place at different frequencies.

The first postulate means that our universe is opened at the macro scale, as well as at the micro scale. At the macro scales means that the event horizon of the universe has different or an unlimited moving boundary depending on the location of the different observers in the universe. This follows directly from Hubble's Law. Also our universe is opened at the micro scale means that elementary particles have no limit on the down scale concept, i.e. quarks have sub- sub- sub- ... structures without lower bound, which is a direct consequence of the phase theory, as will be seen in this paper. Hence CERN represents an intermediate experimental stage, in our life, that cannot go beyond its own. The second postulate is a generalization of the super string theory where the vibrating string is replaced by a 3-dimensional pulsating sink (i.e. opened and closed or widened and narrowed). This postulate also suggests that elementary particles are merely infinitesimal black holes with no lower bound, where aether flows vertically into it. Finally, the third postulate follows directly from the stability of the physical laws.

\section{The pulsating frequency of the elementary particles - the "Heart beat of the Universe"}

Now, what is the pulsating frequency of the elementary particle? To answer this question, it is well known that Plank's constant is used to describe the size of the "Quanta", which is the proportionality constant between the energy $(E)$ of a photon and the frequency, $v$, of its associated electromagnetic wave, viz. $E=h v$. By recalling that, the moment of inertia, $I$ (in SI units is $\mathrm{Kg} \cdot \mathrm{m}^{2}$ ) is a measure of an object's resistance to changes in its rotating or pulsating rate. Then;

$$
\text { Dimensionally; }[\mathrm{h}]=[\mathrm{J} . \mathrm{s}]=\left[\mathrm{ML}^{2} \mathrm{~T}^{-2} . \mathrm{T}\right]=\left[\mathrm{ML}^{2} \mathrm{~T}^{-1}\right]=[\mathrm{I}]\left[\mathrm{T}^{-1}\right]
$$

Thus Plank's constant has the dimension of the moment of inertia multiplied by the frequency. In other words, Plank's constant can be defined as the angular momentum of the elementary particle (the infinitesimal black hole), which describes its rotational (spin) or pulsation state (i.e. quantization). Thus, we can accept the following relationship;

Where $I$ is the moment of inertia of the elementary particle.

$$
\omega=\frac{h}{I} \quad \text { or } \quad f=\frac{\hbar}{I}
$$

So far; we don't know exactly how small quarks and electrons are; they are definitely smaller than $10^{-18}$ meters, and they might literally be points, but still we do not know. By accepting the available dimensions of the electron, and treat it as a (rubber) sphere, then the angular frequency $\omega$, of the spin of the electron (as an infinitesimal black hole) will be constant and equals (Plank's constant / the moment of inertia of the electron). And it is easy to do the following calculations to get a rough estimation of the frequency of the pulsating particles. The moment of inertia of a solid sphere of radius $r$ about its center; $I=(3 / 5) m r^{2}$, the mass of the electron; $m=9.10938215 \times 10^{-31} \mathrm{Kg}$, the radius of the electron; $r_{e}=10^{-19} / 2 \mathrm{~m}$, Plank's constant; $h=6.62606896(33) \times 10^{-34} \mathrm{~J}$.s. Then by direct substitution into eq. (1) will yield; $\omega=4.849 \times$ $10^{35} \mathrm{rad} / \mathrm{sec}$, and the frequency; $f=7.718 \times 10^{34} \mathrm{~Hz}$.

Note: Gamma rays typically have frequencies above 10 exahertz (or $>10^{19} \mathrm{~Hz}$ ), i.e. the pulsating frequency of the elementary particles is far beyond that of Gamma ray frequency. Also note that, cosmic rays are not a form of electromagnetic radiation, but are actually high-energy charged particles with energies similar to, or higher than, the observed gamma electromagnetic radiation energies.

\section{The quantized aether flow}

As seen from the previous articles the aether flows into the elementary particle in quantized form by an angular frequency $\omega$. The flow of aether in the elementary particle can be interpreted as a flow into a sink without lower bound. 


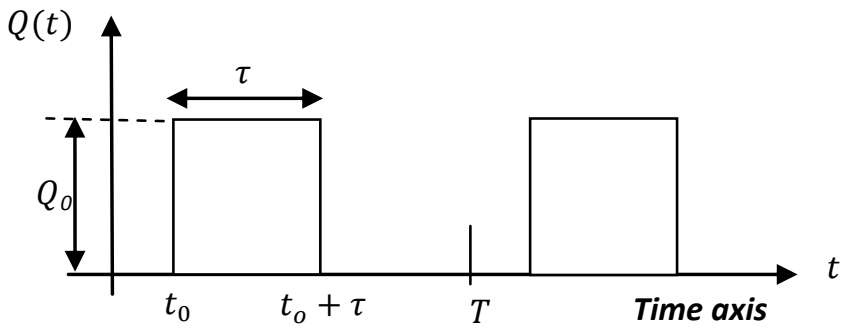

Fig. a: The quantized aether flow $Q(t)$, through an elementary particle to another coincident universe or universes, with time period $T$ and time duration $\tau$.

Fig. a shows two pulses of the vertical aether flow (the discharge) $Q(t)$ into any mass, during a time interval $\tau$ seconds. The flow of aether is taking place through the pulsating elementary particle in the period $T$. We can refer to the aether discharge $Q(t)$, as the sink strength (as the Mass is considered a direct consequence of the aether drag force, and $\left\{Q_{0} * \tau\right\}$ will be referred later as the force carrier). Then $Q(t)$ can be represented mathematically by the following discontinuous eternal function;

$$
Q(t)=\left\{\begin{array}{cr}
0 & 0 \leq t<t_{0} \\
Q_{0} & t_{0}<t<t_{o}+\tau \\
0 & t_{o}+\tau<t \leq T
\end{array}\right.
$$

Then the Fourier series representation of this periodic function will be given by;

$$
Q(t)=\frac{\tau Q_{0}}{T}+\sum_{n=1}^{\infty} \frac{2 Q_{0}}{n \pi} \sin \left(\frac{n \omega}{2} \tau\right) \cos \left[n \omega\left(t-t_{0}-\frac{\tau}{2}\right)\right]
$$

where,

$$
\omega=\frac{2 \pi}{T}
$$

is the angular frequency of the quantized Aether flow rate.

\section{The (stable) force of Interaction between fields can never take place at different frequencies}

As seen from eq. (3), any pulsating particle contains an infinite spectrum of multiple frequencies. Thus any two fields will contain two infinite spectrums of different, but multiple, frequencies. Now, how can the interaction take place between these two fields? It is well known that the force of interaction between any two fields in nature is simply proportional to their products (such as the Poynting Vector). To simplify the analysis, let us consider the two fields given by the following two components;

$$
\begin{gathered}
f_{1}=A_{1} \sin \left(\omega_{1} t+\theta_{1}\right) \text { and } f_{2}=A_{2} \sin \left(\omega_{2} t+\theta_{2}\right) \\
=\left\{\begin{array}{cll}
\text { Average }\left(f_{1} * f_{2}\right) \\
\frac{1}{2}\left(f_{1} \cdot f_{2}\right) \cos \left(\theta_{1}-\theta_{2}\right) & \text { if } & f_{1}=f_{2} \\
\text { indeterminate value or zero } & \text { if } & f_{1} \neq f_{2}
\end{array}\right.
\end{gathered}
$$

Clearly, the (steady state) force of interactions between elementary particles can take place only between particles of the same frequency, as the average value is quite definite and is independent of the frequency itself, hence we use the word "stable". While the average value of the force of interactions between particles with different frequencies are either zero (when both frequencies are not multiple of each other), or indeterminate value and in this case the average value will depend on the elapsed time (the interval) of the interaction, which means chaos or unstable interaction. This interaction could be the one linked with the role of the weak force in the transmutation of quarks that makes the interaction involved in many decays of nuclear particles which require a change of a quark from one flavor to another. Also, $\cos \left(\theta_{1}-\theta_{2}\right)$ is called the power factor of the interactions. It determines solely the type of force of interactions. It is attraction, repulsion or neither depending on the value of the power angle respectively as given;

$$
\left(\theta_{1}-\theta_{2}\right)<\frac{\pi}{2}, \quad\left(\theta_{1}-\theta_{2}\right)>\frac{\pi}{2} \quad \text { or } \quad\left(\theta_{1}-\theta_{2}\right)=\frac{\pi}{2}
$$




\section{The relationship between the Electron Radius and Quarks Radii}

Now, by accepting the fact that, the interaction between elementary particles takes place only at the same frequency and as Planck's constant $h$ is a universal constant, then the dimensions of the quarks, and hence their moments of inertia, should be adjusted to make them have the same angular frequency as the electron. To do that, we should equate the moment of inertia of both the quark and the electron, to get the following relation;

$$
r_{q}=\sqrt{\frac{m_{e}}{m_{q}}} r_{e}
$$

Where, $r_{q}$ and $m_{q}$ are the radius and the mass of the quark respectively; and $r_{e}$ and $m_{e}$ are the radius and mass of the electron respectively. Eq. (8) shows that the radius of the quark, compared to the radius of the electron, is diminished by the square root of the mass of the quark. This formula is in agreement with the concept of the black hole, which shows that the denser the mass of the black hole the smaller will be its radius. According to [3], it is familiar to represent the current quark masses for all 6 flavors as balls of proportional volumes. However, according to the present theory, this is no longer correct as the radii of quarks are inversely proportional to the square root of their masses. Using eq. (8), we can construct table I, which gives the relation between the electron radius and quarks radii. Table I is a sort of prediction of quark radii which are not determined so far. It gives the opportunity to test the phase theory given in this paper, noting that the classical radius of the electron has been used, however the concept is true for any other accepted value for $r_{e}$.

A note about the top quark, the only known way that a top quark can decay, according to the standard model, is through the weak interaction producing a W-boson and a down-type quark (down, strange, or bottom). Because of its enormous mass, the top quark is extremely short-lived with a predicted lifetime of only $5 \times$ $10^{-25} \mathrm{~s}$. As a result the top quarks do not have time to form hadrons before they decay, as other quarks do. This provides physicists with the unique opportunity to study the behavior of a "bare" quark.

Table I: Radii of Quarks versus the Electron Radius

\begin{tabular}{|c|c|c|c|}
\hline Name & Charge & Mass $\left({\left.\mathrm{MeV} / \mathrm{c}^{2}\right)}\right)$ & Radius (Meter) \\
\hline Electron & -1 & 0.511 & $\begin{array}{r}r_{e}=2.8179 \times 10^{-15} \\
\text { (Classical Radius) }\end{array}$ \\
\hline up quark & $+^{2 / 3}$ & $1.7-3.1$ & $(0.5483-0.406) r_{e}$ \\
\hline down quark & $-1 / 3$ & $4.1-5.7$ & $(0.353-0.2994) r_{e}$ \\
\hline charm quark & $+^{2} / 3$ & $1,340-1,180$ & $(0.0195-0.0208) r_{e}$ \\
\hline strange quark & $-1 / 3$ & $130-80$ & $(0.0627-0.0533) r_{e}$ \\
\hline top quark & $+^{2 / 3}$ & $172,900 \pm 600 \pm 900$ & $(0.001716-0.001722) r_{e}$ \\
\hline bottom quark & $-1 / 3$ & $4,370-4,130$ & $(0.0017147-0.0017236) r_{e}$ \\
\hline
\end{tabular}

\section{Note: $1 \mathrm{GeV} / \mathrm{c}^{2}=1.783 \times 10^{-27} \mathrm{~kg}$, Electron Mass $=9.10938188 \times 10^{-31} \mathrm{~kg}$}

\section{The Big Bang equation}

In fact; eq. (8) is the mathematical formulation of the hypothesis of the Big Bang theory, as it can be written in the form;

$$
r_{q}=\frac{U}{\sqrt{m_{q}}}
$$

Where $U$ can be defined as a universal constant, given by $U=\sqrt{m_{e}} r_{e}$, where $m_{e}$ and $r_{e}$ are the mass and the radius of the electron. Clearly; when $m_{q}=\infty, r_{q}=0$, the curvature will be infinite as well as the temperature will be infinite, as the temperature is given by $\left(\mathrm{eV} / \mathrm{k}_{\mathrm{B}}\right.$, where $\mathrm{eV}=1.602 \times 10^{-19} \mathrm{~J}$ and $k_{B}$ is Boltzmann constant $=1.38 \times 10^{-23} \mathrm{~J} / \mathrm{k}$ ). Thus eq. (9) is the singular point of the beginning of the time of creation of the Big Bang theory.

\section{Some Applications; Proton and Neutron}

Consider the fundamental component of eq. (3), $Q_{1}(t)$ where $n=1$, of the vertical aether flow, then we get

$$
Q_{1}(t)=\frac{\tau Q_{0}}{T}+\frac{2 Q_{0}}{\pi} \sin \left(\frac{\omega \tau}{2}\right) \cos \omega\left(t-t_{0}-\frac{\tau}{2}\right)
$$

Using eq. (10), an illustrative example is given in Fig. b, to show the fundamental component of the quantized aether Flow $Q_{1}(t)$, through an elementary particle, which pulsates with an angular frequency $\left(\omega=6.283185, Q_{0}=3, T=1.0 \mathrm{~s}\right.$ and $\left.t_{0}=0.25 \mathrm{~T}\right)$ for different duration times, $\tau(=0.2,0.5,0.8 \mathrm{~s})$. 


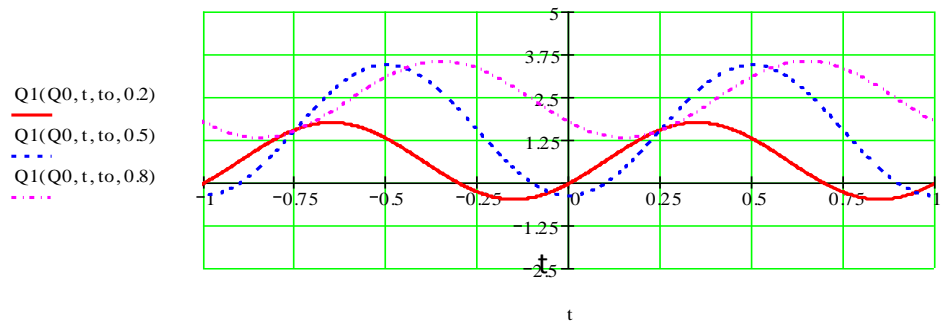

Fig. b: An illustrative example to show the fundamental component $Q_{1}(t)$ of the quantized aether flow rate, through an elementary particle at different time duration $\tau(0.2,0.5,0.8 s)$ where $Q_{0}=3$

Clearly from Fig. b; the effect of changing the duration time, $\tau$, will change the amplitude of the flow, i.e. the discharge of the aether into the elementary particle and hence determines the mass of the elementary particle, as the drag force will be changed. Also, changing the duration time $\tau$ will change the direction of the aether flow rate. Thus, in this example; when the duration time $\tau$ is less than $2 T / \pi(=0.637 T)$ the aether will reverse its direction; i.e. the aether will flow from inside the particle to outside the particle. Thus, the value of the duration time $\tau$ should be greater than $2 T / \pi$ to avoid the aether flow reversing its direction. That is; for unidirectional aether flow, we should have the following condition;

$$
\frac{2}{\pi} T \leq \tau<T
$$

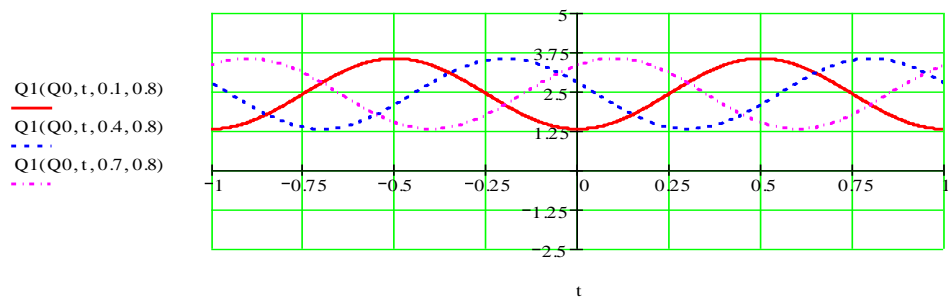

Fig. c: An illustrative example to show the fundamental component $Q_{1}(t)$ of the quantized aether flow through an elementary particle at different starting time $t_{0}(0.1,0.4,0.7 \mathrm{~s})$, where the aether duration time $\tau$ is kept constant and equals $(0.8 s)$.

Similarly, Fig. c shows the effect of changing the starting (or the initial) time $t_{0}$ of the aether pulsating flow. The figure shows the different starting time $t_{0}(=0.1,0.4,0.7 \mathrm{~s})$, where the duration time $\tau$ is kept constant and equals $(0.8 \mathrm{~s})$, where; $Q_{0}=3$, and $T=1.0 \mathrm{~s}$. Clearly, the effect of changing the starting period does not affect the amplitude of the flow, i.e. the discharge (and hence the mass), while it changes the phase of the flow rate.

In the following we shall give illustrative examples to show the mechanism of aether flow through protons and neutrons. Without loss of generality, the demonstration is limited to the fundamental component only, i.e. eq. (10), of the pulsating elementary particle.

\section{Proton}

As we know, proton is composed of two up quarks each of charge $(+2 / 3 \mathrm{e})$, and a down quark of charge $(-1 / 3 e)$, then, by transforming these charges (roughly) to their corresponding flow rate $Q_{0}\left(=2 Q_{0} / 3\right.$, $\left.2 Q_{0} / 3, Q_{0} / 3\right)$, and initial phase time $t_{0}(=2 T / 3,2 T / 3,-T / 3)$. Using eq. (10), then the total aether flow $Q P(t, \tau)$, is simply the sum of the three flow rates of the two up quarks and the down quark, to get

$$
\begin{aligned}
Q P(t, \tau)=\frac{2 \tau Q_{0}}{3 T} & +\frac{4 Q_{0}}{3 \pi} \sin \left(\frac{\omega \tau}{2}\right) \cos \left[\omega\left(t-\frac{2 T}{3}-\frac{\tau}{2}\right)\right]+ \\
& +\frac{2 \tau Q_{0}}{3 T}+\frac{4 Q_{0}}{3 \pi} \sin \left(\frac{\omega \tau}{2}\right) \cos \left[\omega\left(t-\frac{2 T}{3}-\frac{\tau}{2}\right)\right]+ \\
& +\frac{\tau Q_{0}}{3 T}+\frac{2 Q_{0}}{3 \pi} \sin \left(\frac{\omega \tau}{2}\right) \cos \left[\omega\left(t+\frac{T}{3}-\frac{\tau}{2}\right)\right]
\end{aligned}
$$




\section{Neutron}

Similarly, the neutron is composed of two down quarks each of charge $(-1 / 3 e)$, and an up quark of charge $(+2 / 3 e)$. By considering these charges as initial time of pulsations, then on transforming these charges to its time of flow and phase differences, then the total aether flow, $Q N(t, \tau)$, of the neutron is simply the sum of the three flow rates. Then, we get

$$
\begin{aligned}
Q N(t, \tau)=\frac{\tau Q_{0}}{3 T} & +\frac{4 Q_{0}}{3 \pi} \sin \left(\frac{\omega \tau}{2}\right) \cos \left[\omega\left(t+\frac{T}{3}-\frac{\tau}{2}\right)\right]+ \\
& +\frac{\tau Q_{0}}{3 T}+\frac{4 Q_{0}}{3 \pi} \sin \left(\frac{\omega \tau}{2}\right) \cos \left[\omega\left(t+\frac{T}{3}-\frac{\tau}{2}\right)\right]+ \\
& +\frac{2 \tau Q_{0}}{3 T}+\frac{4 Q_{0}}{3 \pi} \sin \left(\frac{\omega \tau}{2}\right) \cos \left[\omega\left(t-\frac{2 T}{3}-\frac{\tau}{2}\right)\right]
\end{aligned}
$$

Plotting eqs. (12) and (13), Fig. $\mathbf{d}$ is drawn using the following parameters; $Q_{0}=3, \tau=0.8, t_{0}=0.25$, $T=1$ and $\omega=6.283$. Fig. $\mathbf{d}$ shows both the proton and the neutron are pulsating, which means that each will have a charge. Of course, this contradicts the fact that, the neutron has no charge, i.e. it should not pulsate.

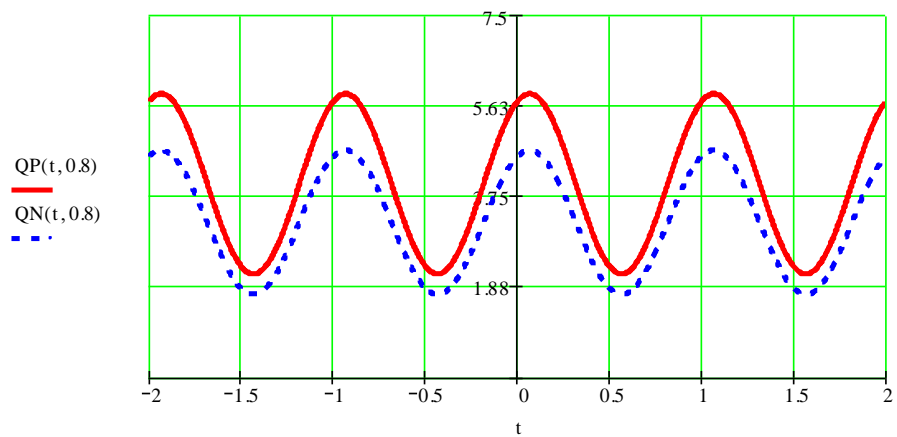

Fig. d: Shows the fundamental component of the quantized aether flow $Q_{1}(t, \tau)$, for both the proton and the neutron.

As we have seen, we limit ourselves to the values of $\tau=0.8$ and $t_{0}=0.25$, which are not the only choice of nature (seek God's Choice).

\section{How can we get a constant flow from a pulsating flow? Hierarchy of quark types and their Sub- Sub- ... structures are direct consequence of the Phase Theory}

It is known that neutron has no charge; this means that aether will flow into it at a constant flow rate not at a pulsating flow rate. The only way to get a constant flow rate from a pulsating flow is that the neutron should be composed of different pulsating particles where their phases are adjusted to get the constant flow rate. The minimum number of particles that satisfy this condition will be, at least three quarks, not two, with appropriate phase angles. Any possibility to use any two quarks will yield two quark-antiquark pair which annihilates each other. Rewrite eq. (10) in the form;

Where;

$$
Q_{1}(t)=\frac{\tau Q_{0}}{T}+\frac{2 Q_{0}}{\pi} \sin \left(\frac{\omega \tau}{2}\right) \cos (\omega t-\theta)
$$

$$
\theta=\omega\left(t_{0}+\frac{\tau}{2}\right)
$$

Eq. (15) is a straight line equation with initial time $t_{0}$ and the duration time $\tau$, for a fixed value $\theta$. This means that, there are infinite numbers of $t_{0}$ and $\tau$ that satisfy eq. (15) for a fixed value of $\theta$. In a similar way as before, the expression of the proton and the neutron are simply the total sum of the aether flow rate through their corresponding quarks, and taking into account the corresponding phases. In this new case, we will choose $\theta$ to be $(2 \pi / 3,2 \pi / 3$ and $-\pi / 3)$ for the proton, and for the neutron $\theta$ will be $(-\pi / 3,-\pi / 3,2 \pi / 3)$. Then the expressions of both proton and neutron will take the forms;

$$
Q_{\text {proton }}(t, \tau)=\frac{2 \tau Q_{0}}{3 T}+\frac{4 Q_{0}}{3 \pi} \sin \left(\frac{\omega \tau}{2}\right) \cos (\omega t-2 \pi / 3)+
$$




$$
\begin{aligned}
+ & \frac{2 \tau Q_{0}}{3 T}+\frac{4 Q_{0}}{3 \pi} \sin \left(\frac{\omega \tau}{2}\right) \cos (\omega t-2 \pi / 3)+ \\
+ & \frac{\tau Q_{0}}{3 T}+\frac{2 Q_{0}}{3 \pi} \sin \left(\frac{\omega \tau}{2}\right) \cos (\omega t+\pi / 3) \\
Q_{\text {neutron }}(t, \tau)=\frac{2 \tau Q_{0}}{3 T} & +\frac{4 Q_{0}}{3 \pi} \sin \left(\frac{\omega \tau}{2}\right) \cos (\omega t+\pi / 3)+ \\
& +\frac{2 \tau Q_{0}}{3 T}+\frac{4 Q_{0}}{3 \pi} \sin \left(\frac{\omega \tau}{2}\right) \cos (\omega t+\pi / 3)+ \\
& +\frac{\tau Q_{0}}{3 T}+\frac{2 Q_{0}}{3 \pi} \sin \left(\frac{\omega \tau}{2}\right) \cos (\omega t-2 \pi / 3)
\end{aligned}
$$

Plotting eq. (16) and eq. (17), using the same parameters given before, $Q_{0}=3, \tau=0.8, t_{0}=0.25, T=$ 1 and $\omega=6.283$. Then, Fig. e shows that the proton pulsates; which means that it has a charge. While the Neutron curve is a straight line parallel to the time axis, i.e. it has no pulsation, which means that it has no charge. It should be noted that the duration time $\tau$ has no influence on the straight line of the neutron, except that it lowers or raises the line, which means that there is only a change in mass, which is a direct consequence of the aether drag force, but it has no charge.

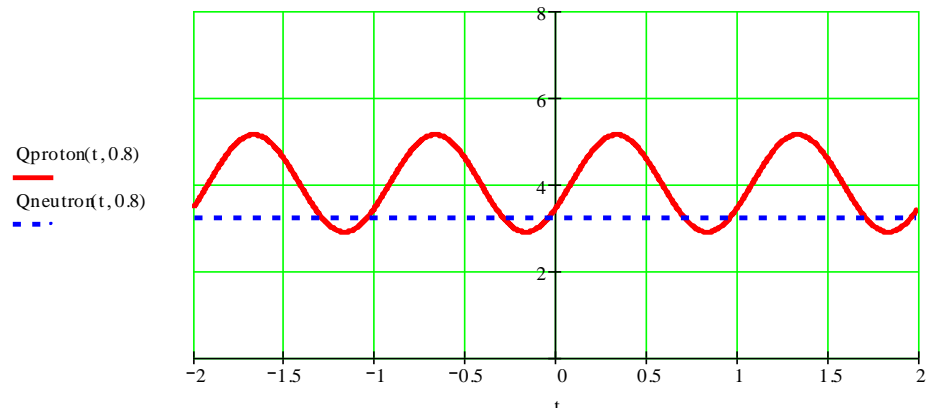

Fig. e: Shows the fundamental component of the quantized aether flow, for both the proton and the neutron. The straight line represents a constant flow, i.e. no pulsation, which means that the neutron has no charge.

Now, although the neutron has no pulsation, its internal structure ( 2 down quarks and one up quark) are pulsating as shown in Fig. f, as followed from eq. (14), with $\theta$ equals $(2 \pi / 3$ and $-\pi / 3)$ and $\tau=0.8$. Thus, the neutron pulsation vanishes due the existence of the phase difference of its 3 different quark components.

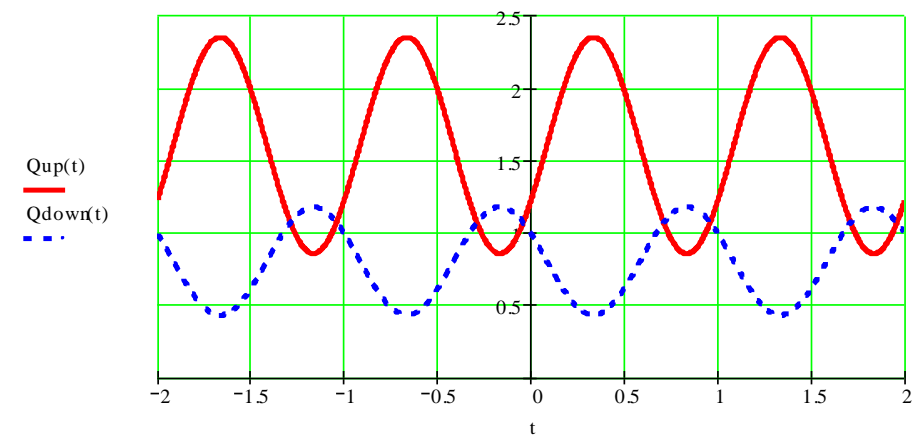

Fig. f: Shows the fundamental component of the quantized aether flow, for both the upper and down quarks.

Finally, there is no reason to prevent extending the definition to include sub- sub- structure of quarks. For example, if the top quark is assumed to be composed of sub-structures, then its first 3 components will be $+4 / 9$, $+4 / 9$ and $-2 / 9$ for the first sub-particles level, and its second 3 sub-structure will be $+8 / 27,+8 / 27$ and $-4 / 27$ for the second sub-particles level, and so on for other components of similar quarks.

\section{Antiparticles}

Antiparticles can easily be interpreted as a particle with a phase shift equals $\pi$ with respect to the phase of its corresponding (or associated) particle. Thus, particles and their antiparticles are completely defined by their 
phase difference $\pi$. Of course it is evident that, when two sine waves, have a phase difference $\pi$, meet together, they will annihilate each other. What is said about electromagnetic interaction can equally hold on the gravitational field or interaction, i.e. the antigravity field is characterized by a phase difference $\pi$ with respect to the phase associated with the gravity field of the particle.

\section{Mass and Force exchange}

In the phase theory we can keep the notion of the force carrier between the interacting particles, but in this case the force carriers will be replaced by merely a "to and fro" motion of the vertical aether flow between elementary particles, i.e. the discharge of the vertical aether flow $\left(\tau Q_{0}\right)$ into the interacting particles, that oscillates between these particles. Fig. g shows a snapshot, where a small particle (left) is closed and lies in the field of an open big particle (right). An infinitesimal time later, the motion is reversed, where the big particle will be closed and lies in the field of the opened small particle, and so on. Clearly, the mass of the elementary particle arises from the aether drag force on each closed particle, and the charge will arise from the pulsation of the elementary particle.

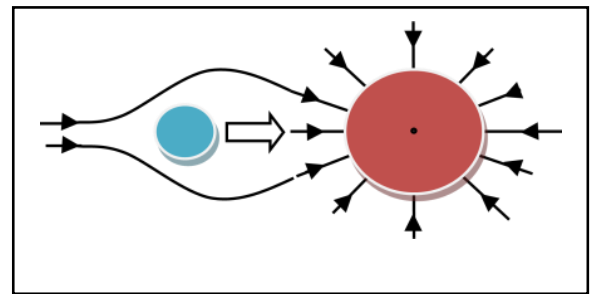

Fig. g: aether drag Force between two elementary particles pulsate with the same frequency but with different phase angles.

Summarizing the situation, each elementary particle has a phase angle for each type of interaction. Thus, the phase theory reduces all the forces of nature to a simple aether motion associated with the corresponding phase angle of each type of interaction.

\section{The Differential Equation of the Super Unified Field Theory (the TOE). Is it a one inch long equation, as some physicists believe, or an infinitely long equation?}

As seen from the previous analyses, each elementary particle is characterized by its four parameters $\left(Q_{o}\right.$, $t_{0}, \tau$ and $\omega$ ). If $n$ is the number of independent particles (the fundamental building blocks) of nature, then the total number of parameters corresponding to these $n$ particles will be $4 n$. To generalize the analysis, let us consider the equation of the theory of everything (the TOE) contains the 4 dimensional parameters $(t, x, y, z)$ and the $4 n$ arbitrary constants defines the 4 associated independent building blocks, and is given in the form;

$$
F\left(t, x, y, z, C_{11}, C_{12}, C_{13}, C_{14}, \ldots \ldots, C_{n 1}, C_{n 2}, C_{n 3}, C_{n 4}\right)=0
$$

To eliminate the $(4 n)$ arbitrary constants, we differentiate eq. (18) successively $4 n$ times with respect to $t$, then the first derivative will be;

$$
\frac{\partial F}{\partial t}+\frac{\partial F}{\partial x} \frac{\partial x}{\partial t}+\frac{\partial F}{\partial y} \frac{\partial y}{\partial t}+\frac{\partial F}{\partial z} \frac{\partial z}{\partial t}=0
$$

Or, in the form of an operator;

$$
\left(\frac{\partial}{\partial t}+\frac{\partial x}{\partial t} \frac{\partial}{\partial x}+\frac{\partial y}{\partial t} \frac{\partial}{\partial y}+\frac{\partial z}{\partial t} \frac{\partial}{\partial z}\right) F=0
$$

Then the $4 n$ derivatives will take the form; 


$$
\left.\begin{array}{c}
\left(\frac{\partial}{\partial t}+\frac{\partial x}{\partial t} \frac{\partial}{\partial x}+\frac{\partial y}{\partial t} \frac{\partial}{\partial y}+\frac{\partial z}{\partial t} \frac{\partial}{\partial z}\right)^{1} F=0 \\
\left(\frac{\partial}{\partial t}+\frac{\partial x}{\partial t} \frac{\partial}{\partial x}+\frac{\partial y}{\partial t} \frac{\partial}{\partial y}+\frac{\partial z}{\partial t} \frac{\partial}{\partial z}\right)^{2} F=0 \\
\vdots \\
\left(\frac{\partial}{\partial t}+\frac{\partial x}{\partial t} \frac{\partial}{\partial x}+\frac{\partial y}{\partial t} \frac{\partial}{\partial y}+\frac{\partial z}{\partial t} \frac{\partial}{\partial z}\right)^{4 n} F=0
\end{array}\right\}
$$

The system of eqs. (21) is (4n) equations; $\left(t, x, y, z, \partial_{x}^{\prime}, \partial_{y}^{\prime}, \partial_{z}^{\prime}, \ldots \ldots \ldots, \partial_{x}^{4 n}, \partial_{y}^{4 n}, \partial_{z}^{4 n}\right) F$, includes the arbitrary constants $\left(C_{11}, C_{12}, C_{13}, C_{14}, \ldots \ldots, C_{n 1}, C_{n 2}, C_{n 3}, C_{n 4}\right)$, which can be solved, in principle, for the $C$ 's. When substituting the values of the $4 n C$ 's parameters into eq. (18), we get a single equation between the variables $\left(t, x, y, z, \partial_{x}^{\prime}, \partial_{y}^{\prime}, \partial_{z}^{\prime}, \ldots \ldots \ldots, \partial_{x}^{4 n}, \partial_{y}^{4 n}, \partial_{z}^{4 n}\right)$, which is the differential equation, of order $4 n$, of the (TOE), which can be written in the form,

$$
\varphi\left(t, x, y, z, \partial_{x}^{\prime}, \partial_{y}^{\prime}, \partial_{z}^{\prime}, \ldots \ldots \ldots, \partial_{x}^{4 n}, \partial_{y}^{4 n}, \partial_{z}^{4 n}\right)=0
$$

From the way of deriving eq. (22), we can say that every function defined by eq. (18) satisfies eq. (22), whatsoever may be the values given to the parameters $\left(C_{11}, C_{12}, C_{13}, C_{14}\right), \ldots \ldots\left(C_{n 1}, C_{n 2}, C_{n 3}, C_{n 4}\right)$. Of course, the function defined by eq. (18) is the particular integral of the differential equation (22). The whole set of particular integrals is the general integral of the same eq. (22). In geometric language, every hyper-surface of eq. (18) is an integral hyper-surface of eq. (22). In other words, eq. (22) is the differential equation of the given family of hyper-surfaces (18). It should be noted that, the above reasoning does not mean that eq. (22) has no integrals other than those presented by eq. (18). The final solution is confirmed by satisfying the boundary conditions of the forces of nature.

One final note; if the number $n$ of the independent particles (i.e. the number of the fundamental building blocks) is infinite, then the length of the differential equation of the (TOE) will be infinite, as the number of its terms is at least greater than $(4 n+1)$, and not one inch long as Michio Kaku and his colleagues believe [1]! Eq. (22) is not only valid for the known elementary particles, but also valid for any number of subsub- ... substructures of the known elementary particles. It is also valid for one level higher than the known elementary particles, i.e. it is still valid at the level of proton, neutron and electron as fundamental building blocks. One final hint, can we know GOD's thought, if we know this equation as Einstein claims [1]? Also, can we use this equation as a life boat to start a new universe, as some physicists believe [1]?! Of course, the answers to these two questions are merely a sort of absurd meaning.

\section{Correlation with the super string theory}

One of the big mistakes in the super string theory is that it considers the different vibrating modes of the string yield different elementary particles. This means, according to article $V$, that the interactions among particles can never take place. Thus, this paper corrects the thought of the super string theory, as it replaces the vibrating string mode by a 3-dimensional infinitesimal pulsating (black) hole mode (like a bubble) where aether is flowing vertically through it. The frequency of pulsation is the same for all particles to assure interactions can take place among them. Different particles are attributed to different phase angles associated with the same frequency, instead of the different frequencies themselves.

\section{Conclusion}

A new theory has been presented, for the first time, called the "Phase Theory", which is the natural evolution of the physical thought and is considered the one beyond the super string theory. In this theory the vibrating string with different frequency modes which defines the different types of elementary particles, is replaced by three dimensional infinitesimal (black) holes that pulsates with the same frequency. Different types of elementary particles are determined by different phase angles associated with the same frequency.

An experimental prediction has been given to confirm the theory presented in the form of the relation between the electron radius and quark radii. The paper also shows that quarks are direct consequence of this theory, and solves "the flavor problem" in QCD, and gives the clue to answer the questions of "Why are there so many flavors?

The direct consequence of the theory presented in this paper is that; charge is due to particle pulsation and mass is due to aether drag force. In short; the theory can be stated in the form: "Based on the vertical aether flow 
into any mass; all the particles of nature have the same pulsating frequency or multiple of it, but each is characterized by its own phase". Thus, the theory can be considered as "The Heart Beat of the Universe".

All figures of the paper are drawn by MathCAD, and a complete numerical data for each figure has been given to make easy check for all calculations made in this paper. Finally we can say that; this paper has put an end to the suffering of mankind in search for the super unifiedfield theory, and its accessible does not mean that our troubles had been ended unless we employ its meaning to know the great creator and understand the objective of our existence.

\section{References}

[1] Isma'eel, M. E.: The Last Chapter of the Symphony of Existence ( $1^{\text {st }}$ edition Publisher: Wahba, Egypt, $2010-2^{\text {nd }}$ edition, Publisher: Author House, UK, 2012).

[2] M. E. Isma'eel*, Sherif M. E. Ismaeel: "Gravitational Blue Shift Confirms the New Phenomenon of the Vertical Aether Flow into any Mass". IOSR Journal of Applied Physics (IOSR-JAP) e-ISSN: 2278-4861. Volume 5, Issue 1 (Nov. - Dec. 2013 ), PP 08-14.

[3] Quark; Wikipedia. http://en.wikipedia.org/wiki/Quark

[4] Superstring theory; Wikipedia. http://en.wikipedia.org/wiki/Superstring_theory 